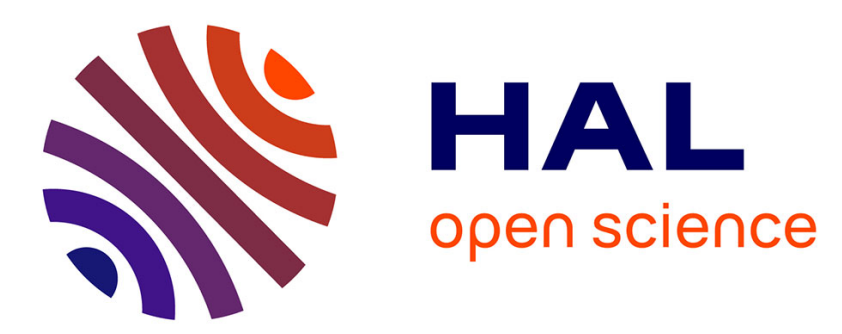

\title{
Impact hydrodynamique bidimensionnel d'un corps parabolique sur une houle d'Airy reguliere
}

\author{
Antoine Morvan, Yves-Marie Scolan
}

\section{To cite this version:}

Antoine Morvan, Yves-Marie Scolan. Impact hydrodynamique bidimensionnel d'un corps parabolique sur une houle d'Airy reguliere. La Houille Blanche - Revue internationale de l'eau, 2017, 103 (6), pp.49 - 56. 10.1051/lhb/2017058 . hal-01784387

\section{HAL Id: hal-01784387 \\ https://hal-ensta-bretagne.archives-ouvertes.fr/hal-01784387}

Submitted on 1 Jun 2021

HAL is a multi-disciplinary open access archive for the deposit and dissemination of scientific research documents, whether they are published or not. The documents may come from teaching and research institutions in France or abroad, or from public or private research centers.
L'archive ouverte pluridisciplinaire HAL, est destinée au dépôt et à la diffusion de documents scientifiques de niveau recherche, publiés ou non, émanant des établissements d'enseignement et de recherche français ou étrangers, des laboratoires publics ou privés. 


\section{La Houille Blanche}

\section{Impact hydrodynamique bidimensionnel d'un corps parabolique sur une houle d'Airy reguliere}

\section{Antoine Morvan \& Yves-Marie Scolan}

To cite this article: Antoine Morvan \& Yves-Marie Scolan (2017) Impact hydrodynamique bidimensionnel d'un corps parabolique sur une houle d'Airy reguliere, La Houille Blanche, 103:6, 49-56, DOI: $10.1051 / \mathrm{lhb} / 2017058$

To link to this article: https://doi.org/10.1051//hb/2017058

\section{(2) Société Hydrotechnique de France, 2017}

曲 Published online: 17 Jan 2018.

Submit your article to this journal $\pi$

Џ Article views: 1

Q View related articles $\sqsubset$

View Crossmark data ¿ 


\title{
Impact hydrodynamique bidimensionnel d'un corps parabolique sur une houle d'Airy reguliere
}

\author{
Antoine MORVAN $^{(1)}$, Yves-Marie SCOLAN $^{(1)}$
}

${ }^{1}$ ENSTA-Bretagne FRE CNRS 3744 IRDL - e-mail : antoine.morvan@ensta-bretagne.org

\begin{abstract}
RÉSUMÉ. - Le problème d'impact hydrodynamique d'un corps parabolique bidimensionnel sur une houle régulière est abordé dans cet article. Le fluide est supposé parfait et incompressible. On se place dans le cadre de la théorie des écoulements potentiels et on résout le problème de Wagner linéarisé. Les champs de pression sont calculés au moyen de la méthode Modified Logvinovich Model (MLM) en négligeant la cinématique du fluide. Plusieurs modèles asymptotiques sont présentées selon que l'impact se produit au voisinage de la crête, du milieu et du creux d'une vague. On présente également un modèle quasi-analytique du problème appelée non-asymptotique. Cette solution permet de prendre en compte toutes les configurations précédentes. Les comportements des différentes configurations sont comparés en termes de corrections mouillées, de champs de pression et d'efforts hydrodynamiques. L'impact d'une parabole sur une surface libre plane est pris en tant que référence. Les résultats confirment que la configuration d'impact engendrant les efforts hydrodynamiques les plus importants est obtenue lorsque l'impact se produit dans le creux d'une vague et lorsque le rayon de la parabole tend vers celui de la vague. Pour des configurations d'impact au voisinage d'une crête et d'un creux, les modèles asymptotiques sont comparés au modèle non-asymptotique. On montre que pour un impact au voisinage d'une crête il n'y a pas de différence entre les deux modèles. Mais lorsque l'impact se produit au niveau d'un creux de légères différences sont obtenues en termes d'efforts. Elles sont dues à la correction de surface mouillée qui est surestimée par le modèle asymptotique à cause de l'approximation faite sur le profil de houle.
\end{abstract}

Mots-clés : hydrodynamique, impact, problème de Wagner, parabole

\section{Hydrodynamic loading of a two-dimensional parabolic body onto a regular Airy wave}

\begin{abstract}
The problem of rigid parabola impact onto a regular Airy wave is considered in this article. The fluid is considered ideal, incompressible and in infinite depth. The liquid flow is assumed to be two-dimensional and potential. The velocity potential induced by the impact of the rigid parabolic body is calculated analytically by means of the Wagner theory. Pressure fields are computed semi-analytically by the Modified Logvinovich Model (MLM) without the fluid kinematics. Different impact models are introduced. Three asymptotic models valid near a previously define point : the crest, the middle and the through. For these three asymptotic models the Airy wave is approximated by a polynomial. Another model is presented, it is not asymptotic and it can take into account all the impact configurations of asymptotic models. For this last model the real shape of the Airy wave is retained. Three asymptotic models are compared for the following physical quantities : the wetted corrections, the pressure fields and the hydrodynamic loads. The case of a parabola impacting on a plane free surface is taken as a reference. It is shown that the configuration generating the more important hydrodynamic loading is obtained when the impact is in the through and when the parabola radius is close to the wave radius. For impact configurations near the crest and the through, asymptotic models are compared to the non-asymptotic model. We show that for an impact near the crest there is no difference between the two models. But when the impact is near the through, slight differences are obtained in terms of slamming coefficient. These differences come from the wetted correction which is overestimated by the asymptotic model. This is due to the polynomial approximation made on the wave profile.
\end{abstract}

Key-words: hydrodynamics, water-entry, slamming, Wagner theory, parabola

\section{INTRODUCTION}

Le tossage engendre des chargements hydrodynamiques locaux conséquents sur les structures flottantes. Le paramètre important est l'angle de relèvement $\beta$ entre la surface libre et la génératrice du corps impactant. Jusqu'à présent, on considérait principalement des surfaces libres planes permettant de caractériser facilement l'influence de ce paramètre. Les travaux ici présentés se concentrent sur l'impact d'un corps solide sur une surface libre non plane et en mouvement.

Von Karman [1929] et Wagner [1932] ont développé des théories qui modélisent les phénomènes d'impact hydrodynamique bidimensionnel en se basant sur la théorie des écoulements potentiels et l'approximation du disque plat. Cette approximation stipule que l'angle de relèvement entre la surface libre et la géométrie du corps impactant demeure faible au cours du temps. Sous cette hypothèse, les conditions limites au niveau de la surface libre et de la surface mouillée du corps sont linéarisées en «aplatissant » les géométries (Fig. 2). Mathématiquement, la surface mouillée du corps impactant se résume à un disque plat dont la dimension horizontale évolue au cours du temps. Au sens de Von Karman, la taille du disque plat est obtenue géométriquement en considérant l'intersection de la surface libre non 
perturbée et de celle corps. Pour Wagner, la taille du disque plat est déterminée en introduisant une condition aux limites supplémentaire (appelée condition de Wagner). À chaque instant cette condition aux limites impose un déplacement fini des particules fluides aux bornes de la surface mouillée. Physiquement, le modèle de Wagner permet de modéliser la remontée locale du fluide le long de la surface mouillée du corps impactant mais pas la formation d'un jet. Il s'agit d'une des limitations majeures de ce modèle qui engendre des champs de pressions et vitesses singulières aux bornes de la surface mouillée. Des améliorations ont été apportées afin d'obtenir un champ de pression borné et continu aux voisinages des limites de la surface mouillée.

On peut citer la méthode des raccordements asymptotiques introduite par Wagner et développée par Zhao et Faltinsen [1993] ainsi qu'Howison et al. [1991]. Elle consiste à raccorder asymptotiquement le champ de pression aux bornes de la surface mouillée au moyen d'une solution locale dans la zone de formation du jet obtenue par transformation conforme. Une autre méthode, initiée par Logvinovich [1969] et développée par Korobkin et Malenica [2005] sous le nom Modified Logvinovich Model s'appuie sur la condition d'imperméabilité exacte. Cette méthode prend en compte les termes quadratiques de l'équation de Bernoulli pour le calcul de la pression. Elle nécessite un développement limité du potentiel des vitesses et l'approximation de ce dernier par le potentiel des vitesses de Wagner.

Une autre version du modèle de Wagner a été développée par Zhao et al. [1997]. Elle s'intitule Generalized Wagner Model, dans ce modèle la condition d'imperméabilité est satisfaite de façon exacte mais les conditions de surface libre sont linéarisées. La résolution du problème est basée sur l'intégration en temps de la condition cinématique de surface libre.

Ces différentes versions du modèle de Wagner sont aussi utilisées pour modéliser des impacts tridimensionnels. Pour une approche analytique numérique on peut citer les travaux de Tassin [2010] reposant sur un couplage type Euler-Lagrange. D'un point de vue analytique Scolan et Korobkin [2001] et Korobkin et Scolan [2006] ont travaillé sur l'impact de corps 3D comme la pyramide. Scolan [2014] a aussi étudié l'impact d'un paraboloïde elliptique au niveau d'une crête de vague.

Le travail présenté dans cet article s'intéresse à ce dernier point. Le problème de l'impact avec présence de houle a été traité dans la littérature, mais essentiellement lorsque l'impact se produit sur une crête de vague. Smith et al. [1998] ont étudié expérimentalement les efforts engendrés par l'impact d'une crête de vague sur une plaque plane. Korobkin et Khabakhpasheva [2006] ainsi que Faltinsen et al. [1997] ont travaillé sur les phénomènes hydroélastiques engendrés par l'impact d'une crête de vague sur une plaque plane déformable.

Plusieurs modèles d'impact hydrodynamique sur une houle d'Airy régulière sont présentés dans cet article. Le point d'impact initial est calculé préalablement. Les différentes configurations d'impact ne se restreignent pas à l'impact au niveau d'une crête, mais à n'importe quel endroit du profil de houle. Les modèles sont comparés au niveau des corrections mouillées, des champs de pression et des efforts hydrodynamiques. Le travail présenté dans cet article utilise une surface libre courbée et en mouvement. La houle est donc assimilée à une surface libre ondulée avançant à une vitesse de phase donnée. Pour ce type de configuration d'impact, on détermine le rôle de l'angle de relèvement $\beta$ en termes de rapport des rayons de courbure au niveau du corps et de la surface libre.

\section{DÉVELOPPEMENTS THÉORIQUES}

L'écoulement induit par l'impact du corps est décrit en théorie des écoulements potentiels. La tension superficielle et la gravité sont négligées. Le profil parabolique $2 \mathrm{D}$ est défini par son rayon de courbure $R$. Il est animé d'une cinématique purement verticale de vitesse $V$. À un instant $t=0 \mathrm{~s}$, il pénètre une surface libre en mouvement. La surface fluide est définie par une houle d'Airy régulière de période $T$, d'amplitude $C$, de pulsation $\omega$ et de phase à l'origine $\theta_{0}$. La houle se propage de la gauche vers la droite. La profondeur d'eau est supposée suffisamment importante pour que la pulsation $\omega$ soit reliée au nombre d'onde $\kappa$ au moyen de la relation de dispersion $\omega^{2}=g \kappa$, avec $g$ l'accélération de la pesanteur.

\section{II.1. Paramétrage géométrique}

À l'instant initial, dans le système de coordonnées galiléen $(0, Y, Z)$ le corps parabolique se situe à une altitude $H_{0}$ et entre en contact avec la houle d'Airy régulière en un point défini par les coordonnées $\left(Y_{0}, Z_{0}\right)$. Dans ce système, la houle et le corps parabolique sont décrits par les équations $1 \mathrm{a}$ et $1 \mathrm{~b}$.

Le point d'impact initial dépend du triplet $\left(Y_{0}, Z_{0}, \theta_{0}\right)$. Pour le déterminer, on résout un système d'équation traduisant l'intersection de la houle et du corps impactant (Eq. 2a) ainsi que l'égalité de leurs pentes respectives en ce point (Eq. 2b).

$$
\begin{aligned}
& \left\{\begin{array}{l}
Z=\frac{Y^{2}}{2 R}+H_{0} \\
Z=C\left(\cos \left(\kappa Y-\omega t+\theta_{0}\right)-1\right)
\end{array}\right. \\
& \left\{\begin{array}{l}
\frac{Y_{0}^{2}}{2 R}+H_{0}=C\left(\cos \left(\kappa Y_{0}+\theta_{0}\right)-1\right) \\
\frac{Y_{0}}{R}=-C \kappa \sin \left(\kappa Y_{0}+\theta_{0}\right)
\end{array}\right.
\end{aligned}
$$

En éliminant les fonctions trigonométriques du système constitué des équations $2 \mathrm{a}$ et $2 \mathrm{~b}$, une équation polynomiale $\mathrm{du} 4^{\mathrm{e}}$ ordre en $Y_{0}$ est obtenue. Une seule racine est retenue et correspond au point d'impact initial lorsque la houle se situe à gauche du corps impactant (voir Fig. 1).

Avec l'expression de $Y_{0}$ retenue, on montre l'unicité du point d'impact initial si et seulement si $R<R_{v}$ avec $R_{v}=\frac{1}{C k^{2}}$.

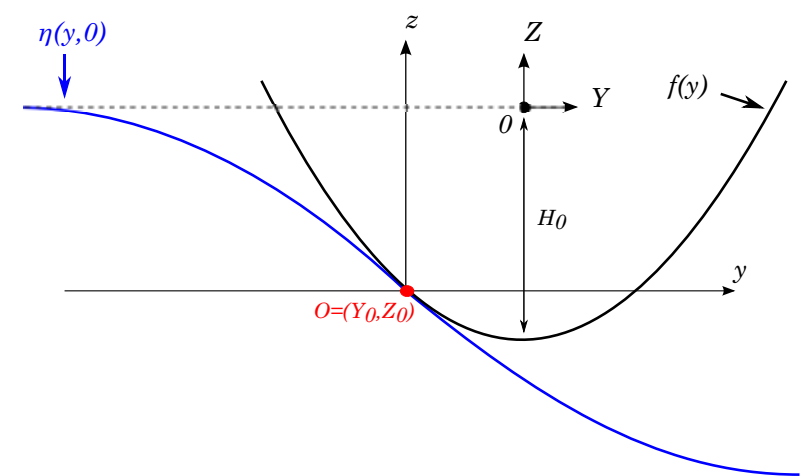

Figure 1 : Configuration initial $t=0 \mathrm{~s}$, les échelles verticale et horizontale ne sont pas identiques

Une fois le triplet $\left(Y_{0}, Z_{0}, \theta_{0}\right)$ déterminé, le problème est centré au niveau du point d'impact initial. Dans le nouveau 
système de coordonnées $(\mathrm{O}, y, z)$, les équations décrivant le corps parabolique et la houle sont :

$$
\left\{\begin{array}{l}
z=f(y)-h(t)=-\frac{Y_{0}^{2}}{2 R}+\frac{\left(y+Y_{0}\right)^{2}}{2 R}-h(t) \\
z=\eta(y, t)=-Z_{0}+C\left[\cos \left(\kappa\left(y+Y_{0}\right)-\omega t+\theta_{0}\right)-1\right]
\end{array}\right.
$$

\section{II.2. Le problème aux limites mixte}

Le problème aux limites mixte linéarisé (Eqs. 4) est formulé au moyen de l'approximation du disque plat. Similairement aux travaux de Korobkin et Puckhnachov [1988], une formulation en termes de potentiel des déplacements $\varphi(y, z, t)$ est privilégiée. Le potentiel des déplacements est défini comme l'intégrale par rapport au temps du potentiel des vitesses. La condition d'imperméabilité permet d'établir la condition de Neumann en déterminant la position relative entre le profil parabolique et la houle d'Airy avec $h(t)=\int_{0}^{t} V(\tau) d \tau$.

Le problème aux limites mixte résultant :

$$
\begin{cases}\Delta \phi=0 & z<0 \\ \phi=0 & z=0, y \in S L(t) \\ \phi_{, z}=-h(t)+f(y)-\eta(y, t) & z=0, y \in S M(t) \\ \phi \rightarrow 0 & \left(y^{2}+z^{2}\right) \rightarrow \infty\end{cases}
$$

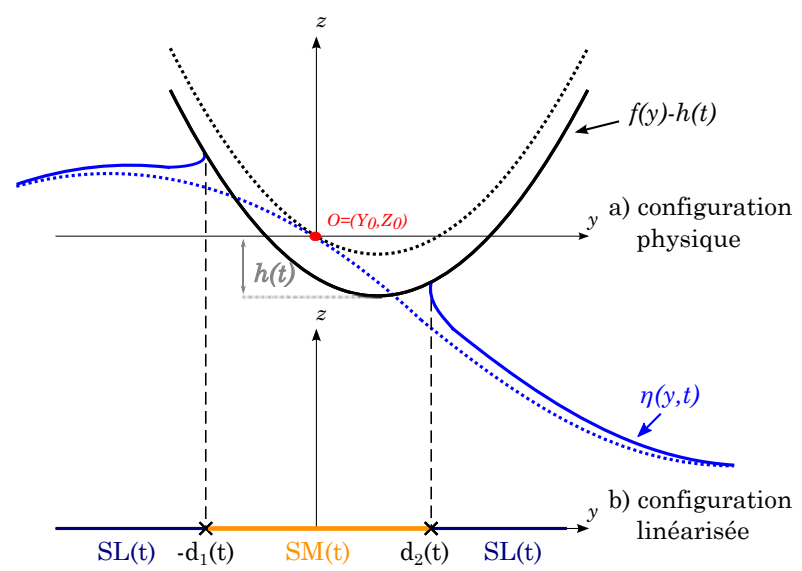

Figure 2 : Schéma de la position relative du corps parabolique après pénétration de la surface libre. Pointillés : position initiale. Trait plein : profil parabolique et géométrie de la surface libre à un instant $t$.

\section{II.3. Résolution du problème aux limites mixte}

Pour simplifier le problème aux limites mixte précédent, trois configurations initiales d'impact sont retenues en fonction de la valeurs de $\left(\kappa Y_{0}+\theta_{0}\right)$ :

- Si l'impact se produit au voisinage de la crête de la vague alors : $\kappa Y_{0}+\theta_{0} \approx 0$

- Si l'impact se produit au voisinage du creux de la vague alors : $\kappa Y_{0}+\theta_{0} \approx \pi$

- Si l'impact se produit au milieu de la pente de la vague alors : $\kappa Y_{0}+\theta_{0} \approx \frac{\pi}{2}$

Les trois configurations d'impact ont les dénominations suivantes : nc (Near Crest), si l'impact se produit au voisinage de la crête, nt (Near Trough) si l'impact se produit au voisinage du creux et $\mathbf{m}$ (Middle) si l'impact se produit au voisinage du milieu de la pente. Conformément à chacune des configurations, un petit paramètre $\epsilon$ est introduit : au voisinage de la crête $\epsilon_{\mathbf{n c}}=\kappa Y_{0}-\omega t+\theta_{0}$, au voisinage du creux $\epsilon_{\mathbf{n t}}=\kappa Y_{0}-\omega t+\theta_{0}-\pi$ et au voisinage du milieu de la pente de la vague $\epsilon_{\mathbf{m}}=\kappa Y_{0}-\omega t+\theta_{0}-\frac{\pi}{2}$. Le paramètre $\epsilon$ dépend donc aussi du temps.

En considérant les $\kappa y$ et $\epsilon$, suffisamment « petits », la condition de Neumann est développée en séries de Taylor pour les trois configurations initiales d'impact. Trois nouvelles conditions de Neumann asymptotiques sont engendrées. À chacune des conditions de Neumann asymptotiques, un modèle d'impact asymptotique est associé.

Un dernier modèle est aussi établi, contrairement aux autres il n'est pas asymptotique. La condition de Neumann complète (Eq. 5) est conservée pour tous les calculs. Ce modèle est donc indépendant de la position initiale d'impact et peut donc prendre en compte l'ensemble des configurations d'impact propres à chacun des modèles asymptotiques. Ce modèle est qualifié de non-asymptotique et sa nomenclature est na. Sa condition de Neumann est la suivante :

$$
\begin{aligned}
\frac{\partial \phi_{\text {na }}}{\partial z} & =-h(t)-\frac{Y_{0}^{2}}{2 R}+\frac{\left(y+Y_{0}\right)^{2}}{2 R}+Z_{0} \\
& -C\left[\cos \left(\kappa\left(y+Y_{0}\right)-\omega t+\theta_{0}\right)-1\right]
\end{aligned}
$$

Pour résoudre les différents problèmes aux limites mixtes, on procède comme dans la littérature existante en remarquant qu'il s'agit de problèmes de type Riemann-Hilbert (voir Gahkov [1990]).

\section{II.3.1. Problèmes symétriques asymptotiques}

Pour les modèles nc et nt, une méthode similaire aux travaux de Scolan [2014] en géométrie tridimensionnelle est utilisée. Cette méthode consiste à développer au second ordre en $\epsilon_{\mathbf{n c}}$ ou $\epsilon_{\mathbf{n t}}$ les conditions de Neumann correspondantes pour faire apparaître la forme canonique suivante :

$$
\frac{\partial \tilde{\phi}}{\partial z}=-\tilde{h}(t)+\frac{\tilde{y}^{2}(t)}{2 \tilde{R}}
$$

Dans le repère mobile $(O, \tilde{y}(\mathrm{t}), z)$ une configuration symétrique d'impact est obtenue. Les potentiels des déplacements et corrections de surface mouillée s'en déduisent facilement :

$$
\left\{\begin{array}{l}
\tilde{\phi}(\tilde{y}, t)=-\frac{1}{6 \tilde{R}}\left(\tilde{a}^{2}-\tilde{y}^{2}\right)^{3 / 2} \text { avec : } \tilde{y}(t)=y-\tilde{c}(t) \tilde{R} \\
\tilde{a}(t)=\sqrt{4 \tilde{R} \tilde{h}}
\end{array}\right.
$$

Les grandeurs tildées ont des expressions distinctes pour les modèles nc et nt, mais ne sont pas présentées ici.

\section{II.3.2. Problème dissymétrique asymptotique}

Pour le modèle d'impact au voisinage du milieu de la pente de la vague, le développement de Taylor au voisinage de son point d'impact initial est réalisé au premier ordre en $\epsilon_{\mathbf{m}}$. La condition de Neumann asymptotique associée à ce modèle est :

$$
\begin{gathered}
\frac{\partial \phi_{\mathbf{m}}}{\partial z}=-h(t)+C \epsilon_{\mathbf{m}}+\frac{y^{2}}{2 R_{\epsilon}}-C \frac{\kappa^{3} y^{3}}{6} \\
\text { Avec }: R_{\epsilon}=\frac{R R_{v}}{R_{v}-\epsilon_{\mathbf{m}} R}
\end{gathered}
$$

La présence d'un terme cubique en y dans l'équation 8 ne permet pas d'avoir une configuration d'impact symétrique dans le plan $(O, y, z)$. Pour déterminer le potentiel 
des déplacements et les corrections de surface mouillée, une formulation d'impact hydrodynamique dissymétrique est utilisée comme dans Scolan et al. [1999].

$$
\begin{gathered}
\phi_{\mathbf{m}}(y, t)=-\frac{1}{12}\left(\frac{2}{R_{\epsilon}}-\frac{3}{2} C \kappa^{3} b-\frac{1}{2} C \kappa^{3} y\right)\left[a^{2}-(y-b)^{2}\right]^{3 / 2} \\
\left\{\begin{array}{l}
-h(t)+C \epsilon_{\boldsymbol{m}}+\frac{b^{2}}{2 R_{\epsilon}}-\frac{C \kappa^{2} b^{3}}{6}+\frac{a^{2}}{4 R_{\epsilon}}-\frac{a^{2} b C \kappa^{3}}{4}=0 \\
\frac{b}{2 R_{\epsilon}}-\frac{b^{2} C \kappa^{3}}{4}-\frac{C \kappa^{3} a^{2}}{16}=0
\end{array}\right. \\
\text { Et : }\left\{\begin{array}{l}
a=\frac{d_{2}+d_{1}}{2} \\
b=\frac{d_{2}-d_{1}}{2}
\end{array}\right.
\end{gathered}
$$

Les équations $11 \mathrm{a}$ et $11 \mathrm{~b}$ sont les corrections mouillées qui traduisent un déplacement fini des particules fluides en $y=-d_{1}$ et $y=d_{2}$.

\section{II.3.3. Problème dissymétrique non-asymptotique}

Le modèle non-asymptotique est aussi traité au moyen de la formulation d'impact hydrodynamique dissymétrique car sa condition de Neumann (Eq. 5) n'est pas symétrique dans le plan $(O, y, z)$. Une décomposition des fonctions trigonométriques en série de fonctions de Bessel $\mathbf{J}_{\mathbf{i}}(a \kappa)$ est nécessaire pour calculer analytiquement le potentiel des déplacements (Eq. 13) et les corrections mouillées (Eqs. 16).

$$
\begin{gathered}
\phi_{\mathbf{n a}}(y, t)=-\frac{1}{6 R}\left[a^{2}-(y-b)^{2}\right]^{3 / 2}-a C\left(A(t) \sum_{k=1}^{\infty} \mathbf{J}_{\mathbf{2}}(a \kappa) f_{k}(y, t)\right. \\
\left.-B(t) \sum_{k=1}^{\infty} \mathbf{J}_{2 \mathbf{k}+\mathbf{1}}(a \kappa) g_{k}(y, t)\right) \\
\left\{\begin{array}{c}
f_{k}(y, t)=\frac{\cos \left[(2 k+1) \arcsin \left(\frac{y-b}{a}\right)\right]}{2 k+1}+\frac{\cos \left[(2 k-1) \arcsin \left(\frac{y-b}{a}\right)\right]}{2 k-1}(14 \mathrm{a}) \\
g_{k}(y, t)=\frac{\sin \left[(2 k+2) \arcsin \left(\frac{y-b}{a}\right)\right]}{2 k+2}+\frac{\sin \left[(2 k) \arcsin \left(\frac{y-b}{a}\right)\right]}{2 k}
\end{array}\right. \\
\left\{\begin{array}{l}
A(t)=\cos \left(\kappa\left(b+Y_{0}\right)-\omega t+\theta_{0}\right) \\
B(t)=\sin \left(\kappa\left(b+Y_{0}\right)-\omega t+\theta_{0}\right)
\end{array}\right. \\
-C(t)+Z_{0}+C+\frac{b^{2}}{2 R}+\frac{b Y_{0}}{4 R} \\
-C \cos \left(\kappa\left(b+Y_{0}\right)-\omega t+\theta_{0}\right) \mathbf{J}_{0}(a \kappa)=0 \\
\frac{a\left(b+Y_{0}\right)}{2 R}+C \sin \left(\kappa\left(b+Y_{0}\right)-\omega t+\theta_{0}\right) \mathbf{J}_{1}(a \kappa)=0
\end{gathered}
$$

Le système d'équations non-linéaires représentant les corrections de surface mouillée (Eqs. 16) du modèle non-asymptotique ne peut, a priori, pas être résolu analytiquement. Les valeurs de $\mathrm{a}$ et $\mathrm{b}$ sont obtenues par une méthode de Newton.

On considère de plus les séries infinies du potentiel des déplacements (Eq. 13) :

$$
\begin{gathered}
F_{k}=\sum_{k=1}^{\infty} \mathbf{J}_{\mathbf{2 k}}(a \kappa) f_{k}(y, t) \\
G_{k}=\sum_{k=1}^{\infty} \mathbf{J}_{\mathbf{2} \mathbf{k}+\mathbf{1}}(a \kappa) g_{k}(y, t)
\end{gathered}
$$

En pratique il s'agit de séries alternées qui convergent très rapidement pour les plages de $a \kappa$ et $y$ considérées dans cette étude. Il a été constaté numériquement que 5 termes sont suffisants.

\section{CHARGEMENT HYDRODYNAMIQUE}

Le champs de pression sont fournis par le modèle dit $M L M$ (Modified Logvinovich Model). La cinématique de houle est comprise dans la condition de Neumann (voir Eq. 4c). Pendant l'impact, la surface libre se comporte comme une tôle ondulée qui se déplace à la vitesse de phase $V_{\varphi}=\frac{\omega}{\kappa}$.

Comme dans les travaux de Scolan [2014], seul le potentiel des vitesses solution du problème aux limites mixte est pris en compte. Dans le cas d'une vitesse constante $V$, la pression $M L M$ est donc similaire à celle détaillée dans les travaux de Korobkin [2004] pour une surface libre plane :

$$
\begin{gathered}
P_{m l m}(y, t)=-\rho\left[\Phi_{, t}+\frac{f^{\prime}(y) V}{1+f_{, y}^{2}} \Phi_{, y}+\frac{1}{2} \frac{\left(\Phi_{, y}^{2}-V^{2}\right)}{1+f_{, y}^{2}}\right] \\
\text { Avec : } \Phi(y, t) \approx \varphi(y, t)-V[f(y)-h(t)]
\end{gathered}
$$

Dans l'équation $20: \varphi(y, t)$ désigne la dérivée par rapport au temps des potentiels des déplacements de la partie II.3. La pression $M L M$ (Eq. 19) fait intervenir des dérivations spatiales et temporelles du potentiel des vitesses qui ne sont pas présentées dans cet article.

\section{COMPARAISONS ET RÉSULTATS}

Différentes configurations physiques sont définies dans le tableau $\mathrm{n}^{\circ} 1$ afin d'étudier l'influence du ratio $R / R_{v}$ pour les modèles d'impact hydrodynamique présentés dans la partie II.3 :

Table 1 : Tableau des configurations physiques d'impact

\begin{tabular}{|c|c|c|c|}
\hline Configurations & $\mathrm{C}$ & $\mathrm{T}$ & $R / R_{v}$ \\
\hline $\mathrm{n}^{\circ} 1$ & 0.0125 & 1.5 & $1 / 25$ \\
\hline $\mathrm{n}^{\circ} 2$ & 0.025 & 1.5 & $/ 12$ \\
\hline $\mathrm{n}^{\circ} 3$ & 0.025 & 1.25 & $1 / 6$ \\
\hline $\mathrm{n}^{\circ} 4$ & 0.05 & 1.25 & $1 / 3$ \\
\hline
\end{tabular}

Dans toutes les configurations présentées ci-dessus, $R=1.0 \mathrm{~m}$ et $V=2.5 \mathrm{~m} / \mathrm{s}$

\section{IV.1. Comparaison des modèles asymptotiques par rapport au modèle d'impact sur une surface libre plane}

Les comparaisons portent sur les efforts hydrodynamiques, les champs de pression et les corrections de surface mouillée. Les efforts hydrodynamiques sont calculés par intégration numérique des champs de pression $M L M$. Ils sont adimensionnés par rapport à l'effort du $1^{\mathrm{e}}$ ordre obtenu pour le cas sans vague. Il a pour expression $F_{\text {wagner }}=2 \rho \pi V^{2} R$. À noter que cet effort est indépendant du temps pour une vitesse $V$ constante. Les champs de pression sont adimensionnés par rapport à la valeur suivante $P_{\max }(t) \simeq \frac{1}{2} \rho \dot{a}_{\text {sans vague }}^{2}(t)$. 


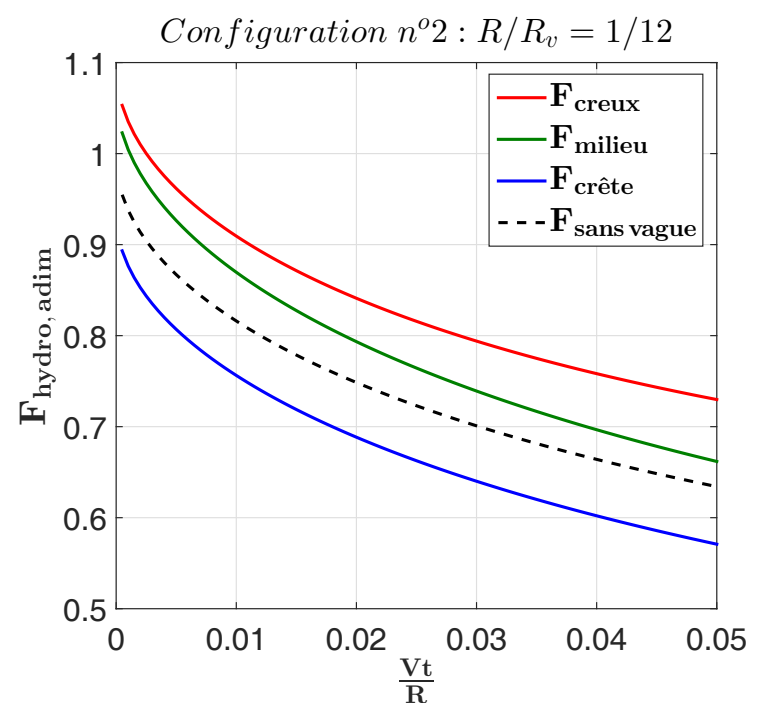

Figure 3 : Comparaisons des efforts hydrodynamiques adimensionnés - config $n^{\circ} 2$

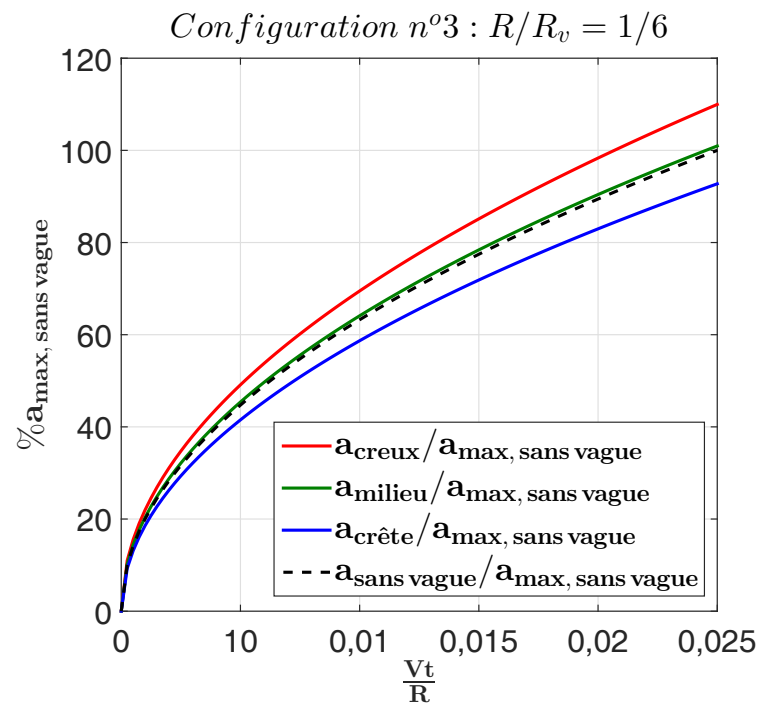

Figure 5 : Comparaisons des corrections mouillées adimensionnées - config $n^{\circ} 3$

D'après les figures 3 et 4 , le modèle qui engendre les efforts hydrodynamiques les plus importants est le modèle d'impact près du creux. Pour la configuration physique $n^{\circ} 3$, il surestime d'environ $20 \%$ le modèle de référence. Le modèle qui présente les efforts hydrodynamiques les plus faibles est le modèle d'impact près de la crête. Il sous-estime d'environ $10 \%$ le modèle de référence. Pour les configurations physiques $\mathrm{n}^{\circ} 2$ et $\mathrm{n}^{\circ} 3$, le modèle d'impact au voisinage du milieu de la pente de la vague surestime d'environ $10 \%$ le modèle de référence. Des tendances similaires sont observées en comparant les corrections de surface mouillée (voir Fig. 5), ainsi que pour les champs de pressions (voir Fig. 6).

Pour les corrections mouillées, les champs de pression et les efforts hydrodynamiques, le modèle de référence se rapproche le plus du modèle d'impact au voisinage du milieu de la pente de la vague.

L'écart entre les différentes grandeurs physiques présentées augmente lorsque le ratio $R / R_{v}$ augmente. Pour les deux configurations physiques testées (voir Tab. 1), les grandeurs physiques calculées avec le modèle d'impact

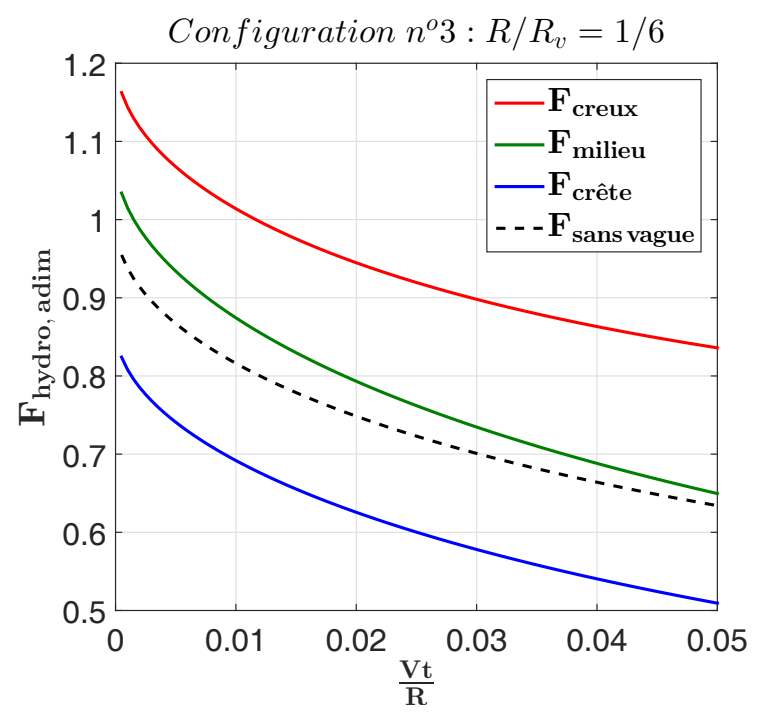

Figure 4 : Comparaisons des efforts hydrodynamiques adimensionnés - config $n^{\circ} 3$

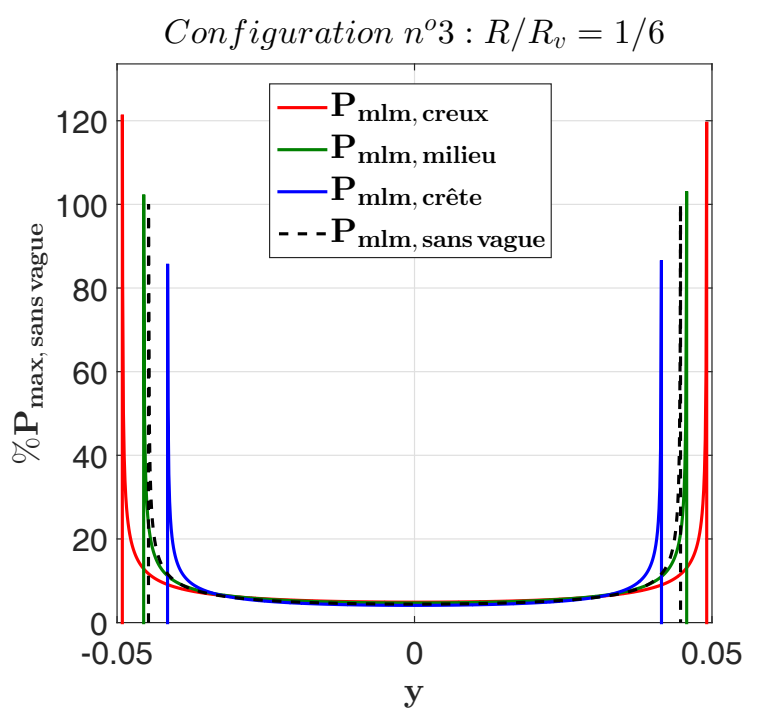

Figure 6 : Comparaisons des champs de pression adimensionnés - config $n^{\circ} 3$. Ici $t=2.0 \mathrm{e}-4 s$

hydrodynamique sur une surface libre plane sont toujours majorées par celles du modèle d'impact au voisinage du creux, et minorées par celles du modèle d'impact au voisinage de la crête.

\section{IV.2. Comparaison avec le modèle non-asymptotique}

Dans cette partie, les modèles asymptotiques (nc et nt) sont comparés au modèle non-asymptotique (na) pour les mêmes points d'impact initiaux près du creux et près de la crête. Les configurations $n^{\circ} 1$, et $n^{\circ} 4$ sont utilisées (voir tableau $n^{\circ} 1$ ). Pour faciliter les comparaisons, deux grandeurs adimensionnées sont introduites :

$$
\begin{gathered}
C_{s}=\frac{F_{\text {hydro }}(t)}{\rho V^{2} R^{2}} \\
C_{p}^{\star}=\frac{P_{m l m}(y, t)}{\frac{1}{2} \rho V^{2}}
\end{gathered}
$$




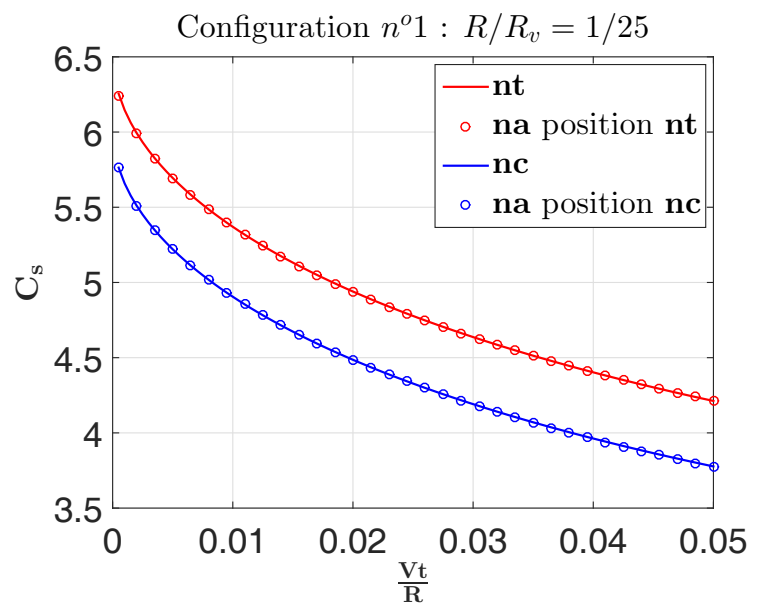

Figure 7 : Coefficients de slamming config. $n^{\circ} 1$

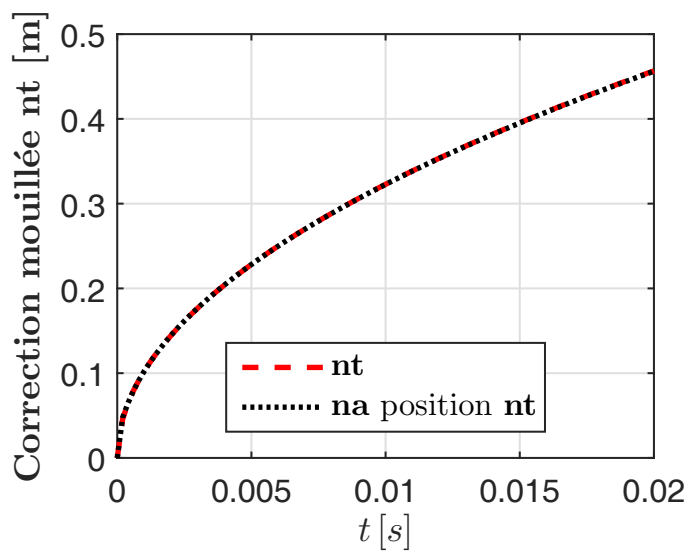

Figure 9 : Corrections mouillées config. $n^{\circ} 1-R / R_{v}=1 / 25$

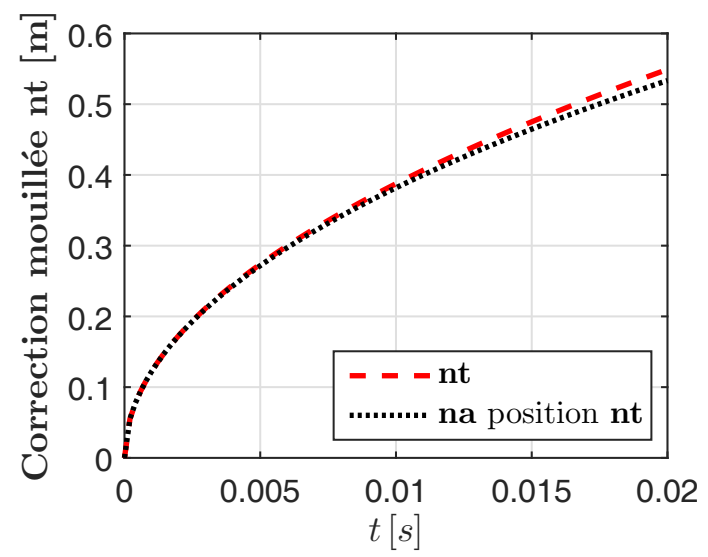

Figure 10 : Corrections mouillées config. $n^{\circ} 4-R / R_{v}=1 / 3$

Les figures 7, 9 et 11 montrent que pour $R / R_{v}=1 / 25$, les résultats pour les coefficients de slamming, les corrections mouillées et les coefficients de pression sont confondus entre les modèles asymptotiques et le modèle non-asymptotique.

Pour un ratio $R / R_{v}=1 / 3$, on constate que le modèle asymptotique d'impact près du creux surestime légèrement au cours du temps le modèle non-asymptotique en terme de coefficient $\mathrm{C}_{\mathrm{s}}$ (voir Fig. 8). Ces différences proviennent

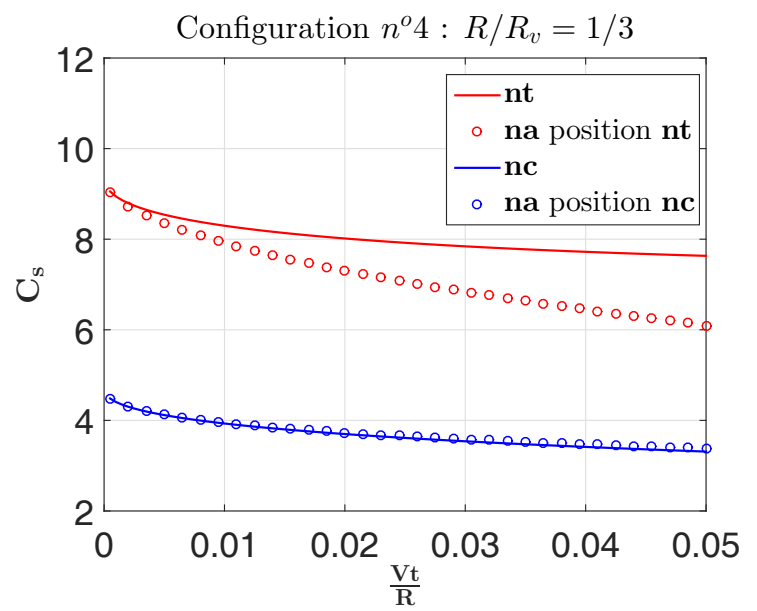

Figure 8 : Coefficients de slamming config. $n^{\circ} 4$
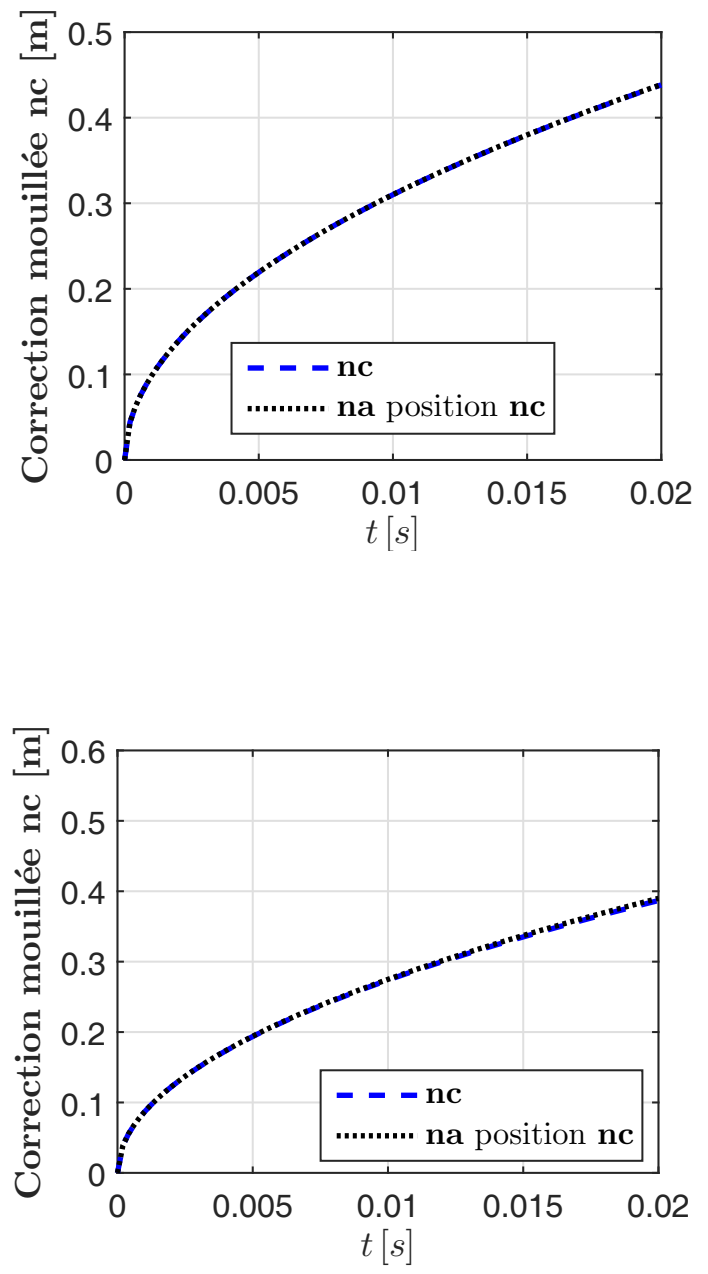

de la surestimation de la correction de surface mouillée du $\mathrm{m}$ (odèle asymptotique pour un impact près du creux (voir Fig. $10 \mathrm{nt}$ ). Les corrections mouillées pour un impact près de la crête entre les modèles asymptotiques et non-asymptotiques sont identiques (voir Fig. 10 nc). Ces observations sont corroborées par les coefficients de pression $C_{p}^{*}$ voir Fig. 12), ils sont confondus pour une position d'impact près de la crête et légèrement différents pour une position d'impact près du creux. 

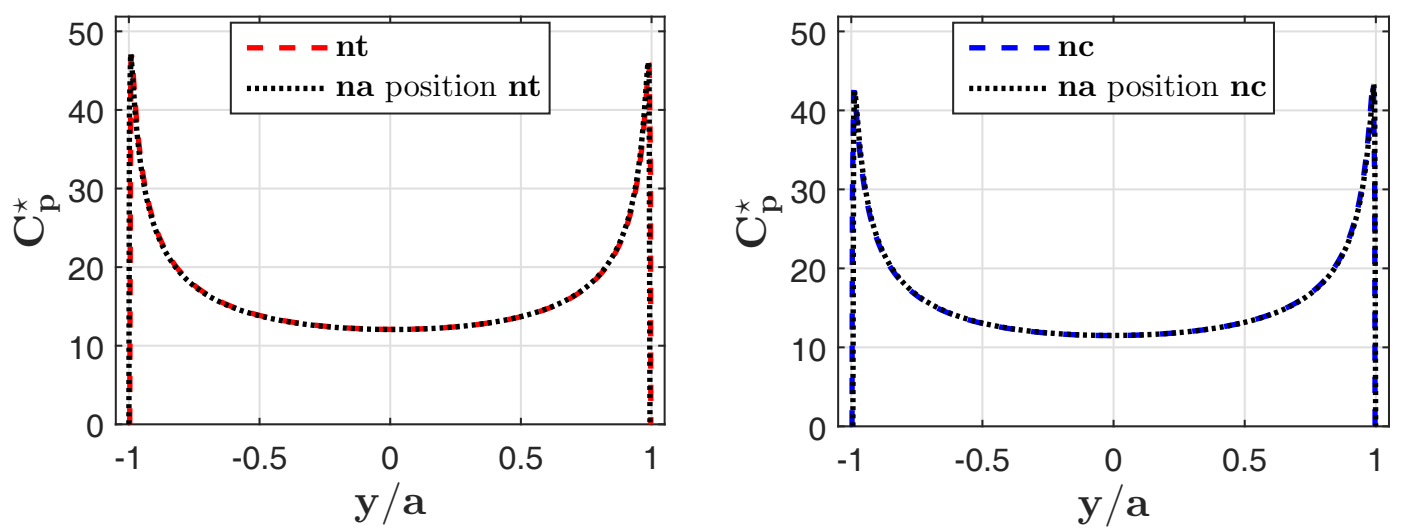

Figure 11 : Coefficient de pression config. $n^{\circ} 1-R / R_{v}=1 / 25$ avec $t=0.01 \mathrm{~s}$
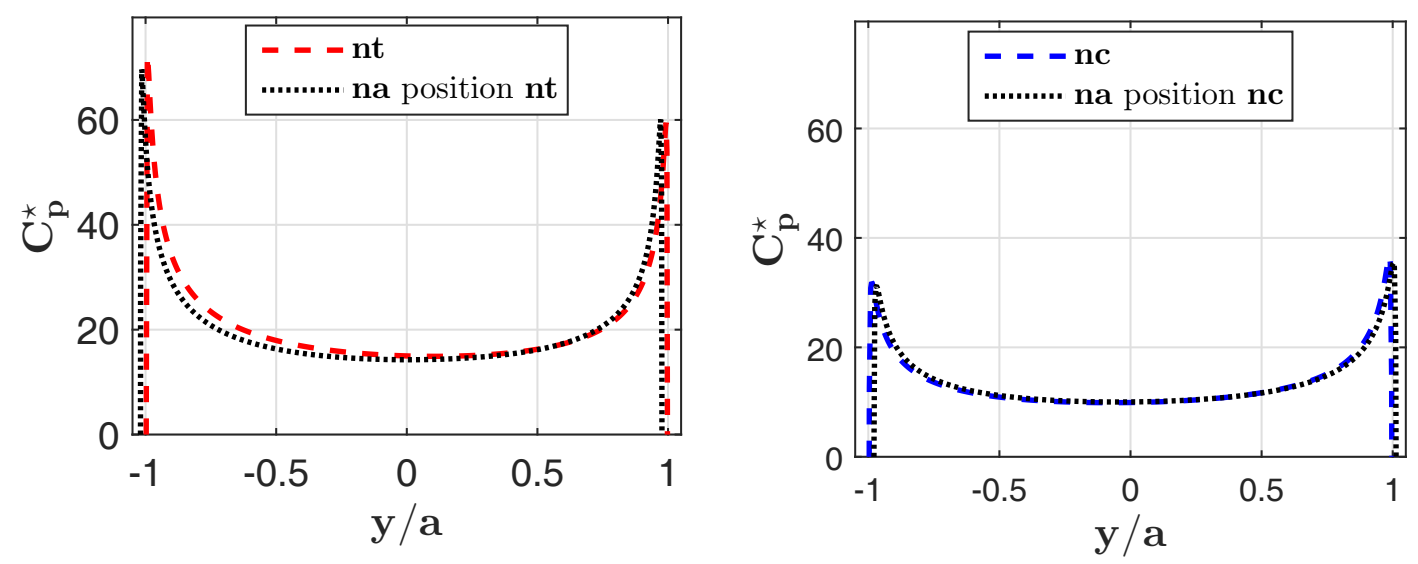

Figure 12 : Coefficient de pression config. $n^{\circ} 4-R / R_{v}=1 / 3$ avec $t=0.01 \mathrm{~s}$

\section{CONCLUSION}

Dans cet article, il est montré qu'il est possible de développer différents types de modèles d'impact hydrodynamique d'un corps parabolique sur une houle d'Airy régulière. On compte, trois modèles asymptotiques valides au voisinage d'un point d'impact initial, ainsi qu'un modèle non-asymptotique qui permet de généraliser les cas particuliers des modèles asymptotiques.

On montre aussi que pour différents ratios $R / R_{v}$, le cas dimensionnant d'impact hydrodynamique d'un corps parabolique n'est pas obtenu lorsque la surface libre est plane et au repos. Mais plutôt lorsque l'impact se produit dans le creux d'une vague.

Il est aussi mis en évidence pour $R / R_{v}=1 / 3$ et pour un impact près du creux que le modèle asymptotique nt surestime le modèle non-asymptotique en termes de coefficient de slamming ( $\approx 10 \%$ en fin de simulation). Cet écart est dû aux corrections de surfaces mouillées. Lorsque $R$ tend vers $R_{v}$ et que les courbures sont du même signe (dans le creux de la vague), le développement à l'ordre 2 du profil de houle d'Airy n'approxime plus correctement le profil de houle réel. La correction mouillée du modèle nt surestime donc celle du modèle na. On conclut que pour un impact dans le creux de la vague avec $R$ du même ordre de grandeurs que $R_{v}$, l'approximation à l'ordre $2 \mathrm{du}$ profil de houle n'est pas suffisante. L'utilisation du modèle non-asymptotique est à privilégier en s'assurant de respecter les hypothèses sur l'angle de relèvement $\beta$. Si $R$ est strictement égal à $R_{v}$ l'angle $\beta$ est égal à zéro. Une configuration d'impact plat sur plat incompatible avec le modèle de Wagner [1932] est atteinte.

\section{REMERCIEMENTS}

Le travail présenté dans cet article est financé par l'ADEME dans le cadre du projet WindKeeper. Ce projet porté par la CNIM consiste à développer un navire innovant dédié à la maintenance des éoliennes offshore. Les membres $\mathrm{du}$ consortium sont la compagnie maritime Chambon, SeaTech Toulon et l'ENSTA-Bretagne.

\section{RÉFÉRENCES}

Faltinsen O.M., Kvålsvold J., Et Aarsnes J.V. (1997) - Wave impact on a horizontal elastic plate. Journal of Applied Marine Science and Technology, 2, 87-100.

F.D. Gahkov. (1990) - Boundary Value Problems. Dover Publications Inc.

Howison S.D., Ockendon J.R., ET Wilson S.K. (1991) Incompressible water-entry problems at small deadrise angles. Journal of Fluids Mechanics, 222, 215-230. 
Korobkin A.A. (2004) - Analytical models of water impact. Journal of Applied Mathematics, 15, 821-838.

Korobin A.A Et Khabakhpasheva T.I. (2006) - Regular wave impact onto an elastic plate. Journal of Engineering Mathematics, 55, 127-150.

Korobkin A.A Et Malenica Š. (2005) - Modified logvinovich model for hydrodynamic loads on asymmetric contours entering water. 20th International Workshop on Water Waves and Floating Bodies, Longyearbyen, Norway.

Korobkin A.A. Eт Puckhnachov V.V. (1988) - Initial stage of water impact. Annual Review Of Fluid Mechanics, 20, 159-185.

Korobkin A.A. Et ScOLAN Y.-M. (2006) - Three-dimensional theory of water impact part 2. linearized wagner problem. Journal of Fluid Mechanics, 549, 343-373.

Logvinovich G. V. (1969) - Hydrodynamics of Flows with Free Boundaries. Naukova Dumka.
SCOLAN Y.-M (2014) - Hydrodynamics impact of an elliptic paraboloid on cylindrical waves. Journal of fluids and Structures, 48, 470-486.

Scolan Y.-M. Et Korobkin A.A. (2001) - Three-dimensional theory of water impact part 1. Inverse Wagner problem. Journal of Fluid Mechanics, 440, 293-326, 2001.

Scolan Y.-M., Coche E., Coudray T., Et Fontaine E. (1999) Etude analytique et numérique de l'impact hydrodynamiques sur des carènes dissymétriques. In. 7ièmes Journées de l'Hydrodynamique.

Smith N.J., Stansby P.K., Et Wright J.R. (1998) - The slam force on a flat plate in free flight due to impact on a wave crest. Journal of Fluids and Structures, 12, 183-196.

TAssin A. (2010) - Modélisation tridimensionnelle d'impacts hydrodynamiques pour l'étude du tossage des bulbes d'étrave. $P h D$ thesis, Université de Bretagne Occidentale. 